\title{
Management of Plasmodium vivax risk and illness in travelers
}

\author{
J. Kevin Baird ${ }^{1,2}$
}

\begin{abstract}
Malaria poses an exceptionally complex problem for providers of travel medicine services. Perceived high risk of exposure during travel typically prompts prescribing protective antimalarial drugs. Suppressive chemoprophylactic agents have dominated strategy for that practice for over 70 years. This broad class of therapeutic agents kills parasites after they emerge from the liver and attempt development in red blood cells. The dominance of suppressive chemoprophylaxis in travel medicine stems largely from the view of Plasmodium falciparum as the utmost threat to the patient - these drugs are poorly suited to preventing Plasmodium vivax and Plasmodium ovale due to inactivity against the latent liver stages of these species not produced by $P$. falciparum. Those hypnozoites awaken to cause multiple clinical attacks called relapses in the months following infection. Causal prophylactic agents kill parasites as they attempt development in hepatic cells. The only drug proven effective for causal prophylaxis against $P$. vivax is primaquine. That drug is not widely recommended for primary prophylaxis for travelers despite preventing both primary attacks of all the plasmodia and relapses of $P$. vivax. The long-held perception of $P$. vivax as causing a benign malaria in part explains the dominance of suppressive chemoprophylaxis strategies poorly suited to its prevention. Recent evidence from both travelers and patients hospitalized in endemic areas reveals $P$. vivax as a pernicious clinical threat capable of progression to severe disease syndromes associated with fatal outcomes. Effective prevention of clinical attacks of vivax malaria following exposure during travel requires primary causal prophylaxis or post-travel presumptive anti-relapse therapy following suppressive prophylaxis.
\end{abstract}

Keywords: Plasmodium vivax, Malaria, Therapy, Prevention, Chemoprophylaxis, Primaquine

\section{Global risk}

The malaria caused by Plasmodium vivax occurs wherever there is Plasmodium falciparum with few exceptions (e.g., Haiti), and sometime occurs as the sole malaria present (e.g., the Korean Peninsula). Although much of Africa has been thought virtually free of $P$. vivax, it is nonetheless relatively common all along the northern Sahel, the Horn, and on Madagascar [1], and travelers to central, western, and southern sub-Saharan Africa acquire $P$. vivax despite dominance of supposedly protective erythrocyte surface Duffy factor negativity in those populations $[1,2]$. Among 618 cases of $P$. vivax in European travelers reported between 1999 and 2003, $21 \%$ were acquired in western, central and southern areas of Africa where $P$. vivax is not prevalent $[1,2]$.

Correspondence: kevin.baird@ndm.ox.ac.uk

${ }^{1}$ Eijkman-Oxford Clinical Research Unit, Jalan Diponegoro No.69, Jakarta 10430, Indonesia

${ }^{2}$ Centre for Tropical Medicine \& Global Health, Nuffield Department of Medicine, University of Oxford, Oxford, UK
Likewise, among 176 repatriated patients diagnosed with $P$. vivax, $32 \%$ acquired the infection in sub-Saharan Africa [3]. Recent evidence suggests $P$. vivax may utilize erythrocyte surface molecules for invasion other than Duffy factor [4]. Transmission without detectable prevalence was suggested by the finding of $13 \%$ of residents of the Congo in west central Africa being positive for antibodies specific to P. vivax sporozoites [5]. About 2.8 billion people live at risk of $P$. vivax with the majority of infections occurring in South and Southeast Asia [6]. This species dominates as the cause of most malaria infections in endemic Central and South America. Virtually everywhere malaria is transmitted, $P$. vivax is present [7] and must be considered in advice to patients visiting those areas.

\section{Virulence}

Appropriate caution demands awareness of $P$. vivax as an often-inherently virulent parasite capable of causing 
severe malaria syndromes associated with death [8]. Although virulence is known to vary with $P$. vivax strains, none occurring today have been demonstrated to rarely progress to severe states of illness. In hospital-based studies from all across the globe, dangerous virulence appears to be common, with severe anemia and thrombocytopenia, respiratory distress, hepatic dysfunction, kidney injury and renal failure, seizures and coma, and circulatory collapse sometime occurring with acute P. vivax malaria [9]. During the 1920 s and 1930 s, patients with neurosyphilis were treated with induced attacks of $P$. vivax malaria, and $5-15 \%$ of otherwise relatively healthy patients did not survive those attacks, most dying at the sixth paroxysm [8]. A meta-analysis of risk of defined severe malaria syndromes among patients living in endemic areas and admitted to hospital with a primary diagnosis of vivax or falciparum malaria showed equal risk between these species $(\mathrm{OR}=0.94$; 95\%CI, $0.2-$ 4.4) [10]. Similar analyses showed slightly lower risk of death with vivax relative to falciparum malaria $(\mathrm{OR}=$ 0.64; 95\%CI, 0.5-0.8) among hospitalized patients [11]. Among patients admitted to American hospitals, those with a diagnosis of $P$. falciparum were more likely to be classified as severely ill relative to $P$. vivax $(\mathrm{OR}=7.7$; 6.3-8.8), but among the severely ill risk of death between the species did not differ significantly $(\mathrm{OR}=1.6$; 0.8-3.2) [12]. These findings also emerged with Plasmodium ovale malaria for severe illness $(\mathrm{OR}=5.0 ; 3.1-8.0)$ and death with severe illness $(\mathrm{OR}=0.84 ; 0.2-3.7)$ relative to $P$. falciparum among those patients treated in the United States [12]. Patients diagnosed with $P$. vivax or $P$. ovale should be considered at risk of progression to severe disease syndromes as dangerous as those associated with a diagnosis of $P$. falciparum.

\section{The relapse problem}

A single infectious bite by an anopheline mosquito bearing sporozoites of $P$. falciparum results in a single attack of malaria, whereas the same bearing $P$. vivax often yields five or more attacks within a year or two. The risk, timing, and number of relapses vary according to origin of infection [13]. Temperate strains and those from the Indian sub-continent are typically less likely to relapse (about 30\%) and do so about 6-8 months following infection, but then also cause multiple attacks ( 3 may be typical). Strains of $P$. vivax from Southeast Asia and Oceania very often ( $>80 \%$ of patients) relapse within 3 weeks of patency of the primary attack and with multiple attacks to follow at approximately 2-month intervals [14]. In two cohorts of Indonesian travelers totaling 1182 people, 382 suffered $P$. vivax attacks within 6 months after return from travel; 80 of those were not treated with primaquine and $79 \%$ relapsed, most within 3 months of patency $[15,16]$. Among 207 P. vivax patients in Thailand treated with chloroquine alone, 79\% had recurrent parasitemia within 2 months [17]. At least some South American strains of $P$. vivax behave similarly [18]. Schwartz and colleagues [19] evaluated 300 cases of malaria among Israeli travelers, and 134 (45\%) occurred more than 2 months following return from travel, with $P$. vivax contributing 129 (96\%) of those attacks. Those authors considered $81 \%$ of those patients as having followed their suppressive chemoprophylaxis regimens in accordance with national guidelines. Travelers provided suppressive chemoprophylaxis and exposed to $P$. vivax but not treated with primaquine at termination of travel face high risk of clinical attacks [20].

\section{Suppressive chemoprophylaxis, PART and G6PD deficiency}

Suppressive chemoprophylaxis does not impact the latent liver forms of $P$. vivax. Protection against late attacks requires presumptive anti-relapse therapy (PART) with primaquine $(0.5 \mathrm{mg} / \mathrm{kg}$ daily for 14 days) after travel. Not all authoritative guidelines recommend PART after travel under suppressive chemoprophylaxis [21-27] and some recommend against it [24]. Among those that do recommend post-travel PART, it is reserved for patients exposed to high risk of $P$. vivax for relatively prolonged periods $[21,26]$. The apparent reluctance to recommend prescribing PART may stem from the relative difficulty of safely and effectively doing so. Some view the 14 daily doses as onerous, and primaquine causes a potentially threatening hemolytic anemia in patients having an inborn deficiency of the enzyme glucose-6-phosphate dehydrogenase (G6PD) [28] necessitating laboratory screening time and costs.

Safe administration of this regimen of primaquine indeed demands affirming G6PD normal status, and adherence to the two weeks of dosing may be viewed as inconvenient or improbable. Nonetheless, if patients and providers alike understand the potentially threatening nature of acute attacks by $P$. vivax, G6PD screening and good adherence may be viewed as less problematic than with a supposedly benign and not threatening illness. Further, recently available point-of-care G6PD diagnostics may ease the inconvenience and cost of that screening [29]. The U.S. Food and Drug Administration approved at least one such screening kit [30].

Users of qualitative G6PD screening kits of any type should be aware of the insensitivity to G6PD levels above $30 \%$ of normal enzyme activity [31]. This often occurs among female heterozygotes for this X-linked trait having red blood cell populations mosaic for the deficiency at any frequency between 0 and $100 \%$ due to the phenomenon of lyonization during embryonic development. Females screened as "normal" may thus be at risk of up to $70 \%$ hemolytic loss of red blood cells with 
primaquine dosing [11]. Female travelers may require a quantitative laboratory assessment of G6PD status. There is no firmly established quantitative cut off of G6PD activity for safety with primaquine, but $>70 \%$ of normal would exclude most heterozygotes and be unlikely to seriously threaten those included.

\section{Causal chemoprophylaxis}

One of the most widely prescribed drugs for malaria chemoprophylaxis is Malarone ${ }^{\circ}$ (GlaxoSmithKline UK, atovaquone-proguanil). The atovaquone component of this formulation showed causal prophylactic activity against $P$. falciparum, i.e., killed developing liver stages [32]. However, this activity may be absent or weak against $P$. vivax liver stages [33, 34], although some evidence suggests otherwise [35]. This important unresolved question requires clarification. Primaquine is the only available drug proven to arrest the development of both primary tissue schizonts and hypnozoites [36]. When taken as $0.5 \mathrm{mg} / \mathrm{kg}$ daily during exposure to infectious anophelines, primaquine prevented primary attacks of both $P$. falciparum and $P$. vivax with good efficacy $(>90 \%)$, in addition to preventing the formation of hypnozoites of $P$. vivax [36, 37].

Confusion regarding the term causal prophylaxis merits some clarification here. Some authorities explain that causal prophylaxis impacts only developing tissue schizonts in the liver and does not impact hypnozoites [24]. While it is true that some drugs can prevent the formation of hypnozoites while being unable to kill formed hypnozoites [38], in practice causal prophylaxis begins in travelers free of infection. In the specific instance of primaquine as causal prophylaxis, it prevents the formation of both tissue schizonts and hypnozoites, like the experimental drug PI4 kinase [38]. It is thus incorrect to infer that causal prophylactics will not prevent the seeding of the liver with hypnozoites. Primaquine prophylaxis, unlike PI4 kinase [38], also happens to kill formed hypnozoites in patients infected without causal prophylaxis, i.e., as post-suppressive prophylaxis PART or radical cure of acute vivax malaria.

Primaquine as primary causal prophylaxis offers several distinct advantages. No pre-travel dosing is necessary, and post-travel dosing of a few days suffices. The regimen of course also abrogates the need for post-travel PART. These attributes give primaquine chemoprophylaxis great appeal for travelers departing on short notice or only briefly exposed to risk (e.g., a week or so) whom may otherwise endure several weeks of pre- and posttravel dosing with a suppressive chemoprophylactic and PART. Nonetheless, if $P$. vivax indeed occurs wherever there is malaria risk, and the clinical necessity of preventing relapses may be accepted, then primaquine as primary prophylaxis may be considered as a viable option for any traveler, regardless of destination or duration of exposure. The clinical trials of primaquine prophylaxis dosed subjects daily for 3 to 12 months with good safety and tolerance (when administered with a snack or meal) and efficacy against $P$. vivax and $P$. falciparum [36].

The principal shortcomings with primaquine for primary prophylaxis include the necessity of daily dosing, G6PD screening, contraindications with pregnancy or infancy, and emerging evidence of cytochrome P-450 isozyme 2D6 (CYP2D6) polymorphisms rendering primaquine inactive in some patients $[39,40]$. The frequency of CYP2D6 alleles associated with therapeutic failure of primaquine is not known and varies widely among ethnic populations, but $1-10 \%$ may be a reasoned forecast [41]. Complete assurance of primaquine activity may require ascertaining CYP2D6 genotype, a relatively expensive and sophisticated laboratory analysis with a complex and nuanced clinical interpretation. Another potential pitfall with primaquine is its lack of activity against the blood stages of $P$. falciparum, despite good activity against the same of $P$. vivax. The traveller experiencing a breakthrough of $P$. falciparum may not be protected by on-going primaquine prophylaxis, whereas a suppressive prophylactic regimen would perhaps slow progression of the infection despite failure. Finally, the label of primaquine does not include an indication for primary prophylaxis, necessitating offlabel prescribing.

Travel medicine experts informing guidance on malaria chemoprophylaxis have not put primaquine as primary causal prophylaxis forward as a recommended option except as a last resort or for special circumstances (Table 1). Some of the pitfalls listed above likely weighed in that deliberation. Acknowledging that no regimen of chemoprophylaxis is without risk and potential pitfalls, the provider weighs the relevant risks and benefits to each traveler in deciding upon appropriate protection. That weighing should include primary attacks and relapses of $P$. vivax as a potentially serious threat to travelers visiting almost any malarious area. Vivax malaria should not be considered a benign and inconsequential risk. Primaquine as primary causal prophylaxis or as PART following suppressive prophylaxis effectively deals with the problem of $P$. vivax relapses after travel. Current guidelines deal poorly with preventing those by the uniform primacy of suppressive chemoprophylaxis strategy and weakness of recommendations regarding post-travel PART in that strategy.

\section{Ideal chemoprophylaxis}

The ideal drug for preventing malaria in travelers would exert both causal and suppressive activity with weekly or longer interval dosing, not threaten G6PD deficient or 
Table 1 Authoritative recommendations regarding primaquine for the prevention and treatment of $P$. vivax malaria

\begin{tabular}{|c|c|c|c|c|c|}
\hline & United States & & United Kingdom & Canada & International \\
\hline Source & U.S. government & The Medical Letter & U.K. government & Canadian government & World Health Organization \\
\hline $\begin{array}{l}\text { Primaquine as } \\
\text { Primary Prophylaxis }\end{array}$ & $\begin{array}{l}\text { As an alternative to } \\
\text { suppressive options or: } \\
\text { With travel where } \\
\text { malaria is primarily } \\
\text { P. vivax } \\
\text { Short-term travel } \\
\text { Short-notice travel }\end{array}$ & $\begin{array}{l}\text { Only as an alternative } \\
\text { to suppressive options }\end{array}$ & Not recommended & $\begin{array}{l}\text { Only as an alternative } \\
\text { to suppressive options }\end{array}$ & $\begin{array}{l}\text { None specified except for special } \\
\text { risk groups (infants, pregnant } \\
\text { women, vulnerable children in } \\
\text { seasonal high transmission) }\end{array}$ \\
\hline $\begin{array}{l}\text { Primaquine following } \\
\text { a diagnosis of } P \text {. vivax }\end{array}$ & Recommended & Recommended & Recommended & Recommended & Strongly recommended \\
\hline $\begin{array}{l}\text { Primaquine following } \\
\text { suppressive prophylaxis }\end{array}$ & Recommended & No recommendation & Not recommended & $\begin{array}{l}\text { Recommended for } \\
\text { high risk groups }\end{array}$ & No recommendation \\
\hline References & [21] & {$[22,23]$} & {$[24,25]$} & [26] & [27] \\
\hline
\end{tabular}

pregnant patients, not be reliant upon appropriate cytochrome P-450 phenotypes for good activity, and include the indication in its approved label. No such drug is currently available, although one in clinical development may more closely approach this ideal than any others now available. Tafenoquine is a primaquine-like 8-aminoquinoline under development by GlaxoSmithKline (GSK, UK) and the Medicines for Malaria Venture (MMV, Geneva) for radical cure of $P$. vivax [42]. Earlier, the drug had been under development by GSK and the U.S. Army as a chemoprophylactic agent with good efficacy against $P$. falciparum and $P$. vivax when administered monthly for up to 5 months [43]. Onset of a mild reversible vortex keratopathy occurred in most subjects of another trial dosed weekly for 6 months [44]. Tafenoquine shares the hemolytic toxicity problem of primaquine [45] and its single dose with relatively long elimination half-life elevates the importance of robust G6PD screening in conjunction with intended clinical use. Dependence upon CYP2D6 phenotypes for activity is not yet known for tafenoquine. Despite these known or potential pitfalls, should the development of tafenoquine for chemoprophylaxis resume and result in a labeled indication, it may well offer a superior option for travelers exposed to $P$. vivax and $P$. falciparum malarias.

\section{Radical cure of Plasmodium vivax}

Patients diagnosed with malaria caused by $P$. vivax require therapy that terminates the acute attack (blood schizontocidal) and prevents subsequent attacks by relapse (hypnozoitocidal). This treatment is called radical cure and several options for it may be considered [46]. Many different regimens of primaquine therapy have been recommended over the 60 years of its availability as the only hypnozoitocide, but the $0.5 \mathrm{mg} /$ $\mathrm{kg}$ daily for 14 days is now widely considered preferred among those [27, 47]. Many nations recommend $0.25 \mathrm{mg} / \mathrm{kg}$ daily for 14 days, but likely do so despite relatively low efficacy [48] in order to mitigate risk of harm due to poor access to G6PD screening.
Chloroquine-resistant $P$. vivax occurs globally but is most common in areas of Southeast Asia and Oceania [49]. Treatment of those infections necessitates use of quinine or artemisinin-combined therapies (ACTs) with primaquine for radical cure.

Although some authorities include a recommendation for artemether-lumefantrine with primaquine for radical cure of chloroquine-resistant $P$. vivax, only dihydroartemisininpiperaquine and artesunate-pyronaridine combined with primaquine have been demonstrated as safe, well tolerated and efficacious for radical cure in G6PD-normal nonpregnant subjects $[15,16]$. The dependence of primaquine activity on CYP2D6 metabolism renders it vulnerable to impeded efficacy by partner drugs that may inhibit CYP2D6 activity. Lumefantrine happens to be a moderately potent CYP2D6 inhibitor and The Medical Letter [23] explicitly warns against use of artemether-lumefantrine with drugs that are metabolized by CYP2D6.

Evidence-based medical practice demands proof of safety, tolerability, and efficacy with drugs used in tandem, as in radical cure of $P$. vivax malaria. Blood schizontocides may not be responsibly combined with primaquine without such evidence [50]. The same will be true the hypnozoitocide tafenoquine following its aspired availability in practice. A single dose of tafenoquine combined with chloroquine for radical cure has shown good safety, tolerability, and efficacy in G6PD-normal non-pregnant research subjects infected by $P$. vivax [42]. The label of tafenoquine would thus be very likely to narrowly specify chloroquine as the partner blood schizontocide. Given the rise of chloroquine-resistant $P$. vivax, tafenoquine would inevitably be combined with artemisinin-combined therapies. Doing so responsibly will require demonstrations of safety, tolerability, and efficacy for each partner blood schizontocide in radical cure.

\section{Conclusions}

Malaria caused by $P$. vivax occurs wherever malaria is transmitted with very few exceptions. Despite long being 
perceived as an intrinsically benign species, evidence now affirms this species as capable of progressing to severe disease syndromes associated with fatal outcomes, including in travelers. Effective prevention of $P$. vivax in travelers is not achieved with the suppressive chemoprophylaxis strategies that dominate authoritative guidance on preventing malaria in travelers. The latent hypnozoites of this species are not affected by suppressive chemoprophylaxis, and unless the exposed traveler is prescribed post-exposure PART with primaquine, late attacks by $P$. vivax may occur. Primary causal chemoprophylaxis using daily primaquine $(0.5 \mathrm{mg} / \mathrm{kg})$ during exposure prevents primary attacks of $P$. falciparum and $P$. vivax, and prevents relapses by the latter with good safety, tolerability, and efficacy in G6PD normal nonpregnant subjects (>90\%), despite likely failures due to relatively infrequent CYP2D6 inadequate metabolizer phenotypes. Providers of travel medicine services should consider the advantages and pitfalls of both suppressive and causal prophylactic strategies in preventing attacks of $P$. vivax now firmly linked to risk of poor clinical outcomes. Both strategies apply primaquine, either as PART or primary prophylaxis, respectively, and necessitates coping with risk to G6PD-deficient or pregnant patients. Likewise, travelers suffering acute attacks of $P$. vivax should be considered at high risk of multiple recurrences if they do not receive radical cure employing primaquine, the only therapeutic option at present. In all facets of responsibly managing risk and illness of $P$. vivax in travelers, primaquine is involved and providers have to deal with the serious problem of its toxicity with G6PD deficiency. This will remain especially true following the anticipated registration and availability of tafenoquine as a single dose (with chloroquine) agent for radical cure of $P$. vivax malaria.

\section{Abbreviations}

ACT: Artemisinin combined therapy; CYP2D6: Cytochrome P-450 isozyme 2D6; G6PD: Glucose-6-phosphate dehydrogenase; PART: Presumptive anti-relapse treatment

\section{Acknowledgements}

JKB is grateful to the editorial staff of BMC Tropical Diseases, Travel Medicine and Vaccines for inviting this article.

\section{Funding}

JKB is supported by the Wellcome Trust Major Overseas Program Viet Nam grant.

\section{Availability of data and materials}

Not applicable.

\section{Author's contributions}

JKB wrote this review without co-authors.

\section{Authors' information}

JKB trained in microbiology, protozoology, parasitology, biochemistry, and epidemiology at the University of Maryland (B.SC., 1980; M.Sc., 1984) and Tulane University (Ph.D., 1994). He served 22 years on active duty in the US Navy Medical Service Corps as a malaria specialist with duties in Indonesia, the Philippines, Vietnam, Thailand, Cambodia, Vanuatu, Guyana, Peru, and Ghana. He is currently Professor of Malariology in the Centre for Tropical Medicine \& Global Health, Nuffield Department of Medicine, University of Oxford, Oxford, United Kingdom, and directs the Eijkman-Oxford Clinical Research Unit at the Eijkman Institute of Molecular Biology in Jakarta, Indonesia. JKB and his colleagues in the US Navy conducted some of the pioneering work on primaquine chemoprophylaxis during the 1990s and early 2000s. He has conducted multiple clinical trials evaluating that and other chemoprophylactic agents against malaria, and is considered expert at the diagnosis, treatment, prevention and control of human malaria, particularly those involving $P$. vivax. He routinely advises national malaria control programs, industry partners, civil society, and the World Health Organization on these technical issues.

\section{Competing interests}

JKB receives research support from GlaxoSmithKline UK for the conduct of a clinical trial of tafenoquine for radical cure of $P$. vivax malaria.

\section{Consent for publication}

Not applicable.

Ethics approval and consent to participate Not applicable.

\section{Publisher's Note}

Springer Nature remains neutral with regard to jurisdictional claims in published maps and institutional affiliations.

Received: 16 November 2016 Accepted: 17 March 2017

Published online: 28 March 2017

\section{References}

1. Howes RE, Reiner Jr RC, Battle KE, et al. Plasmodium vivax transmission in Africa. PLoS Negl Trop Dis. 2015;9:e0004222.

2. Mühlberger N, Jelinek T, Gascon J, et al. Epidemiology and clinical features of vivax malaria imported to Europe: sentinel surveillance data from TropNetEurop. Malaria J. 2004;3:5.

3. Elliot JH, O'Brien D, Leder $\mathrm{K}$, et al. Imported Plasmodium vivax malaria: demographic and clinical features in nonimmune travelers. J Travel Med. 2004;11:213-9.

4. Menard D, Barnadas C, Bouchier C, et al. Plasmodium vivax clinical malaria is commonly observed in Duffy-negative Malagasy people. Proc Natl Acad Sci U S A. 2010;107:5967-71

5. Culleton $\mathrm{R}$, Ndounga $\mathrm{M}$, Zeyrek FY, et al. Evidence for transmission of Plasmodium vivax in the Republic of the Congo, west central Africa. J Infect Dis. 2009:200:1465-9.

6. Guerra CA, Howes RE, Patil AP, et al. The international limits and population at risk of Plasmodium vivax transmission in 2009. PLoS Negl Trop Dis. 2010:4:e774

7. Howes RE, Battle KE, Mendis KN, et al. Global epidemiology of Plasmodium vivax. Am J Trop Med Hyg 2016; 95(supplement 6): in press.

8. Baird JK. Evidence and implications of mortality associated with acute Plasmodium vivax malaria. Clin Microbiol Rev. 2013;26:36-57.

9. Anstey NM, Douglas NM, Poespoprodjo JR, Price RN. Plasmodium vivax: clinical spectrum, risk factors, and pathogenesis. Adv Parasitol. 2012;80:151-201.

10. Naing C, Whittaker MA, Nyunt Wai V, Mak JW. Is Plasmodium vivax malaria a severe malaria? A systematic review and meta-analysis. PLoS Negl Trop Dis. 2014;8:e3071.

11. World Health Organization. Point-of-care G6PD testing to support safe use of primaquine for the treatment of vivax malaria. WHO Evidence Review Group meeting report, 8-9 October 2014, WHO/UNAIDS Building, Geneva, Switzerland. WHO 2015. www.who.int/malaria/mpac/mpac-march2015-ergg6pd.pdf. Accessed 27 Mar 2017.

12. Hwang J, Cullen KA, Kachur SP, Arugin PM, Baird JK. Severe morbidity and mortality risk from malaria in the United States, 1985-2011. Open Forum Infect Dis. 2014;1:ofu034.

13. Battle KE, Karhunen MS, Bhatt S, et al. Geographical variation in Plasmodium vivax relapse. Malaria J. 2014;13:144.

14. White NJ. Determinants of relapse periodicity in Plasmodium vivax malaria. Malaria J. 2011;10:297. 
15. Sutanto I, Tjahjono B, Basri $\mathrm{H}$, et al. Randomized, open-label trial of primaquine against vivax malaria relapse in Indonesia. Antimicrob Agents Chemother. 2013;57:1128-35.

16. Nelwan EJ, Ekawati LL, Tjahjono B, et al. Randomized trial of primaquine hypnozoitocidal efficacy when administered with artemisinin-combined blood schizontocides for radical cure of Plasmodium vivax in Indonesia. BMC Med. 2015;13:294

17. Aung PP, Lwin KM, Price RN, et al. Dihydroartemisinin-piperaquine versus chloroquine in the treatment of Plasmodium vivax malaria in Thailand: a randomized controlled trial. Clin Infect Dis. 2011;53:977-84.

18. Hanf M, Stephani A, Basurko C, Nacher M, Carme B. Determination of the Plasmodium vivax relapse pattern in Campoi, French Guiana. Malaria J. 2009:8:278.

19. Schwartz E, Parise M, Kozarsky P, Cetron M. Delayed onset of malaria - implications for chemoprophylaxis in travelers. New Engl J Med. 2003;349:1510-6.

20. Chu CS, White NJ. Management of relapsing Plasmodium vivax malaria. Expert Rev Infect Ther. 2016;14:885-900.

21. United States Centers for Disease Control. The Yellow Book 2016. https:// wwwnc.cdc.gov/travel/yellowbook/2016/infectious-diseases-related-totravel/malaria\#1939. Accessed 26 Feb 2017.

22. The Medical Letter on Drugs and Therapeutics. Advice for Travelers. 2015;57(1466):52-8.

23. The Medical Letter on Drugs and Therapeutics. Drugs Parasitic Infect. 2013; 11 (Suppl):e1-31.

24. Public Health England. Guidelines for Malaria Prevention in Travelers from the United Kingdom 2016. Crown copyright 2017; 120.

25. Lalloo DG, Shingadia D, Pasvol G, et al. UK malaria treatment guidelines. J Infection. 2007;54:111-21.

26. Public Health Agency of Canada. Canadian Recommendations for the Prevention and Treatment of Malaria. 2014. p. 135.

27. World Health Organization. Guidelines for the Treatment of Malaria. 3rd ed. 2015. p. 316.

28. Ashley EA, Recht J, White NJ. Primaquine: the risks and the benefits. Malaria J. 2014;13:418.

29. Oo NN, Bancone G, Maw LZ, et al. Validation of G6PD point-of-care tests among healthy volunteers in Yangon, Myanmar. PLoS ONE. 2016;11:e0152304.

30. Osorio L, Carter N, Arthur P, et al. Performance of the BinaxNOW G6PD deficiency point-of-care diagnostic in P. vivax infected subjects. Am J Trop Med Hyg. 2015;92:22-7.

31. Baird JK, Dewi M, Subekti D, et al. Noninferiority of glucose-6-phosphate dehydrogenase deficiency diagnosis by a point-of-care rapid test vs the laboratory fluorescent spot test demonstrated by copper inhibition in normal human red blood cells. Transl Res. 2015;165:677-88.

32. Berman JD, Nielsen $R$, Chulay JD, et al. Causal prophylactic activity of atovaquone-proguanil (Malarone) in a human challenge model. Trans Roy Soc Trop Med Hyg. 2001;95:429-32.

33. Jimenez BC, Navarro M, Huerga $\mathrm{H}$, et al. Tertian malaria (Plasmodium vivax and Plasmodium ovale) in two travelers despite atovaquone-proguanil prophylaxis. J Travel Med. 2006;13:373-5.

34. Maguire JD, Llewellyn DM. Relapsing vivax malaria after 6 months of daily atovaquone/proguanil in Afghanistan: the case for expanded use of primaquine as a causal prophylactic. J Travel Med. 2007;14:411-4.

35. Mavrogordato A, Lever AML. A cluster of Plasmodium vivax malaria in an expedition group to Ethiopia: prophylactic efficacy of atovaquone/proguanil on liver stages of P. vivax. J Infect. 2012;65:269-74.

36. Alving AS, Rucker $\mathrm{K}$, Flanagan $\mathrm{CL}$, et al. Observations on primaquine in the prophylaxis and cure of vivax malaria. In: Proceedings of the $6^{\text {th }}$ International Congress of Tropical Medicine and Malaria, vol. 7. 1959. p. 203-9.

37. Baird JK, Fryauff DJ, Hoffman SL. Primaquine for prevention of malaria in travelers. Clin Infect Dis. 2003;37:1659-67.

38. Zeeman AM, Lakshminarayana SB, van der Werff N, et al. PI4 kinase is a prophylactic but not radical curative target in Plasmodium vivax-type malaria parasites. Antimicrob Agents Chemother. 2016:60:2858-63.

39. Bennett JW, Pybus BS, Yadava A, et al. Primaquine failure and cytochrome P-450 2D6 in Plasmodium vivax malaria. N Engl J Med. 2013:369:1381-2.

40. Ingram RJ, Crenna-Darusallam C, Soebianto S, Noviyanti R, Baird JK. The clinical and public health problem of relapse despite primaquine therapy: case review of repeated relapses of Plasmodium vivax acquired in New Guinea. Malaria J. 2014;13:488.
41. Gaedigk A, Sangkuhl K, Whirl-Carillo M, Klein T, Leeder JS. Prediction of CYP2D6 phenotype from genotype across world populations. Genet Med. 2016. doi:10.1038/glm.so16.80.

42. Llanos-Cuentas A, Lacerda MV, Rueangweerayut $R$, et al. Tafenoquine plus chloroquine for the treatment and relapse prevention of Plasmodium vivax malaria: a multicentre, double-blind, randomised, phase $2 b$ dose-selection study. Lancet. 2014;383:1049-58.

43. Walsh DS, Eamsila C, Sasiprapha T, et al. Efficacy of monthly tafenoquine for prophylaxis of Plasmodium vivax and multi-drug resistant $P$. falciparum malaria. J Inf Dis. 2004;190:1456-63.

44. Nasveld PE, Edstein MD, Reid M, et al. Randomized, double-blind study of the safety, tolerability, and efficacy of tafenoquine versus mefloquine for malaria prophylaxis in non-immune subjects. Antimicrob Agents Chemother. 2010;54:792-8.

45. Shanks GD, Oloo AJ, Aleman GM, et al. A new primaquine analog tafenoquine (WR238605), for prophylaxis against Plasmodium falciparum malaria. Clin Infect Dis. 2001:33:1968-74.

46. Baird JK, Valecha N, Duparc S, White NJ, Price RN. Diagnosis and treatment of Plasmodium vivax malaria. Am J Trop Med Hyg. 2016;95 Suppl 6:35-51.

47. Hill DR, Baird JK, Parise ME, Lewis LS, Ryan ET, Magill AJ. Primaquine: report from CDC expert meeting on malaria chemoprophylaxis I. Am J Trop Med Hyg. 2006:75:402-15.

48. John GK, Douglas NM, Von Seidlein $L$, et al. Primaquine radical cure of Plasmodium vivax: a critical review of the literature. Malaria J. 2012:11:280.

49. Price RN, Von Seidlein L, Valecha N, et al. Global extent of chloroquineresistant Plasmodium vivax: a systematic review and meta-analysis. Lancet Infect Dis. 2014;14:982-91.

50. Baird JK. Resistance to chloroquine unhinges vivax malaria therapeutics. Antimicrob Agents Chemother. 2011;55:1827-30.

\section{Submit your next manuscript to BioMed Central and we will help you at every step:}

- We accept pre-submission inquiries

- Our selector tool helps you to find the most relevant journal

- We provide round the clock customer support

- Convenient online submission

- Thorough peer review

- Inclusion in PubMed and all major indexing services

- Maximum visibility for your research

Submit your manuscript at www.biomedcentral.com/submit 